\title{
De la invisibilidad de la situación de las policlínicas comunitarias-rurales en Uruguay a la priorización de la salud rural como política pública.
}

\author{
From the invisibility of the situation of rural and community health services in Uruguay \\ towards the prioritization of rural health as Public Policy.
}

Fernando Borgia', Alexis Gularte², Ingrid Gabrielzyk ${ }^{3}$, Marcela Azambuja4, Jorge Soto5, Miguel Corneo ${ }^{6}$, Helena Giménez , Marlene Arraras, Sebastián González

${ }^{1}$ Magister en Sociología. Director de la investigación. Docente invitado en cursos de grado y posgrado (Uruguay y América Latina). Ex Asesor de la División Salud y actualmente es parte del Centro de Formación y Estudios de la Intendencia de Montevideo. Coordinador de la Región Cono Sur de la Asociación Latinoamericana de Medicina Social (ALAMES), integrante del Movimiento de Salud de los Pueblos. fernando.borgia@gmail.com

2 Psicólogo Social y Psicodramatista. Integrante de la Dirección Nacional de ALAMES. Integrante del equipo de coordinación de Salud Sexual y Reproductiva y fortalecimiento de la Red de Atención Primaria (RAP) de la Administración de Servicios de Salud del Estado (ASSE) del departamento de Salto. ale.jgc@gmail.com

${ }^{3}$ Lic. En Trabajo Social, Docente en el Departamento de Enfermería Comunitaria Facultad de Enfermería Universidad de la República, Maestría en Primer Nivel de Atención - en etapa de tesis, Asesora en Programa ITS/NIH-SIDA del Ministerio de Salud Pública (MSP); integrante de ALAMES. ingrid.gabrielzyk@gmail.com

${ }^{4}$ Lic. En Psicología., Referente del Instituto Nacional de las Mujeres (INMujeres) del Ministerio de Desarrollo Social (MIDES) para el departamento de Salto. Coordinadora Adjunta de la Red de Género de ALAMES. marceaza@gmail.com

${ }_{5}^{5}$ Psicólogo Social y Psicodramatista. Ex Coordinador Técnico de la Oficina de la Mujer y la de la Intendencia de Salto. Integrante del Equipo de Formación de Recursos Humanos y de Salud Sexual y Reproductiva de la ASSE en el departamento de Salto. Coordinador Nacional de ALAMES. jsotoviera@gmail.com

${ }^{6}$ Integrante de la Dirección de EMAUS Maldonado, Mediador Comunitario integrante de la Mesa Intersocial del departamento de Maldonado, integrante de la Dirección Nacional de ALAMES.

Agente Comunitaria en Salud, Mediadora Comunitaria, representante del Movimiento Nacional de Usuarios de Servicios de Salud Pública y Privada (MNUSPP) en la Junta Departamental de Salud, integrante de EMAUS y de ALAMES.

${ }^{8}$ Maestra Inspectora. Integrante de ALAMES.

Estudiante de Sociología. Integrante de ALAMES.
RESUMEN La creación del Sistema Nacional Integrado de Salud (SNIS) pretendió revertir la fragmentación e inequidad preexistente en el acceso, oportunidad y calidad de la atención a la salud. Su implementación progresiva priorizó la incorporación de prestadores integrales de servicios de salud que brindan servicios en los tres niveles de complejidad de atención. Propósito: visibilizar la situación de las policlínicas comunitarias y rurales no incorporadas al SNIS, su historia y generar propuestas-acciones de incidencia para su integración al SNIS y el desarrollo de la Atención Primaria de Salud Integral (APS-I). Métodos: Investigación-Acción-Participativa, cuantitativos, cualitativos, y análisis colectivo con población. Conclusiones: se contribuyó a la desinvisibilización de la situación de las policlínicas comunitarias y rurales, y a la priorización de la salud rural como política pública.

PALABRAS CLAVE: Salud de población rural; Atención Primaria en Salud; equidad en salud; sistemas de salud.

ABSTRACT The creation of the National Integrated Health System (NIHS) attempted to reverse the existing fragmentation and inequity in access, opportunity and quality of health care. Progressive implementation incorporating prioritized comprehensive providers of health services, which provide services at the three levels of complexity of care. Purpose: To visualize the situation of rural and community health services unincorporated to NIHS, its history and generate action-proposals, advocacy for integration into the NIHS and the development of Comprehensive Primary Health Care (CPHC). Methods: Participatory-Action-Research, quantitative, qualitative, and collective analysis with participants. Conclusions: contribution to desinvisibilization of the community and rural health services situation, prioritization of rural health as public policy.

KEYWORDS: Rural health; Primary health care; Health equity; Health systems 


\section{Introducción}

A partir de la asunción del gobierno por el Frente Amplio (FA), en el año 2005, el Estado reconoce la salud como derecho y como bien público. La creación, en el año 2007, del Sistema Nacional Integrado de Salud (SNIS), pretendió revertir la fragmentación e inequidad preexistente en el acceso, oportunidad y calidad de la atención a la salud. Su implementación progresiva 'priorizó la incorporación de prestadores integrales', que brindan servicios en todos los niveles de complejidad de atención. En la estructura de la organización sanitaria se reconocen dos subsectores: el público y el privado, cuya conformación puede apreciarse en detalle en el gráfico 1. El sector privado concentraba en el ańo 2005 el $75 \%$ del gasto, por lo que el consumo por usuario de las Instituciones de Asistencia Médica Colectiva (IAMC) es dos veces y media superior al de la Administración de Servicios de Salud del Estado (ASSE), existiendo una importante correlación entre tipo de cobertura, nivel de ingresos y actividad, y resultando en una 'salud para ricos' y una 'salud para pobres'. Además, dado que la población que recurre a los servicios públicos de salud tiene avanzada edad, la inequidad es también mayor, resultando en que - al comenzar la investigación - el sistema gastaba más en quienes tenían menores necesidades de atención en salud.

Con la Ley 17.930 de Presupuesto Nacional (2005) se introducen los primeros cambios jurídicos relevantes, orientados a la reforma sanitaria nacional, pues "se diseña el Presupuesto del Ministerio de Salud Pública (MSP) con énfasis en la construcción del SNIS y del Seguro Nacional de Salud (SNS), el aumento de los salarios más sumergidos, el fortalecimiento del Primer Nivel de Atención, la reconstrucción de la carrera funcional, la reestructuración del sistema de remuneraciones, y la descentralización de la ASSE" (MUÑOZ, 2006). En el año 2007, el Parlamento aprobó dos leyes fundamentales que facilitarán el análisis y la comprensión de la propuesta de reforma sanitaria: Ley 18.131 de creación del Fondo Nacional de Salud (FONASA), Ley 18.211 "reglamentaria del derecho a la protección de la salud que tienen todos los habitantes residentes en el país" y establece "las modalidades para el acceso a prestaciones integrales del SNIS", y su decreto reglamentario de 2008. Los principios que rigen el SNIS se resumen en el gráfico 2.

La reforma en curso implica tres cambios complementarios y progresivos (BORGIA, 2005): cambio de modelo de atención, cambio de modelo de gestión y cambio de modelo de financiamiento (gráfico 2). El proceso de reforma en su actual fase de desarrollo implica la coexistencia parcial con las modalidades pre-existentes de cobertura asistencial de la salud, por lo que 'la no integración actual al SNIS no significa que la persona no tenga cobertura asistencial'. La incorporación al SNIS implica una 'única e integral prestación' brindada por el prestador elegido (entre los prestadores integrales), por el/la usuario/a, con una cápita ${ }^{1}$ establecida, con un plus ${ }^{12}$ por el cumplimiento de metas prestacionales (vinculadas a la prevención y promoción de la salud), un pago ${ }^{13}$ variable en función de un porcentaje de los ingresos laborales y la integración del núcleo familiar, y copagos variables dependiendo del prestador elegido.

El SNIS no incluye a los prestadores parciales (gráfico 2). Estos fueron los que más se desarrollaron por la vía organizacional/sindical y/o comunitaria (gráfico 1), en intentos por 'dar alguna respuesta sanitaria' a los trabajadores (formales o informales) de un gremio, o a los miembros de una comunidad que por sus características (dimensión, lejanía, pobreza, capacidad organizativa y de resistencia a la dictadura, etc.) no tenían respuestas del Estado ni del Mercado. Muchos de estos servicios coexisten aún hoy bajo la modalidad de Policlínicas Comunitarias y/o Servicios de Salud Sindicales o de Cajas de Auxilio. Cuando tuvo lugar el auge neoliberal en el país, el aumento de los precios de las coberturas integrales tradicionales llevó al desarrollo de otro tipo de

\footnotetext{
Valor económico de la cobertura asistencial integral, regulado por el Estado.

${ }^{2}$ Valor económico de un pago adicional a la cápita, variable según la meta de que se trate, regulado por el Estado.

${ }^{3}$ Este aspecto es fundamental porque desvincula la necesidad asistencial de la capacidad de pago, es decir, 'se paga de acuerdo a la capacidad'y se 'utiliza de acuerdo a la necesidad'.

${ }^{4}$ Pagos de bolsillo que debe hacer el/la usuario/a en el momento de demandar atención. Estos tienen valores variables dependiendo del prestador y el Estado establece los montos máximos legales. En los servicios de ASSE (subsector público) no se exigen copagos para ningún/a usuario/a que haya elegido a ASSE como su prestador.
} 


\section{Gráfico 1. Sistema de salud en Uruguay (1)}

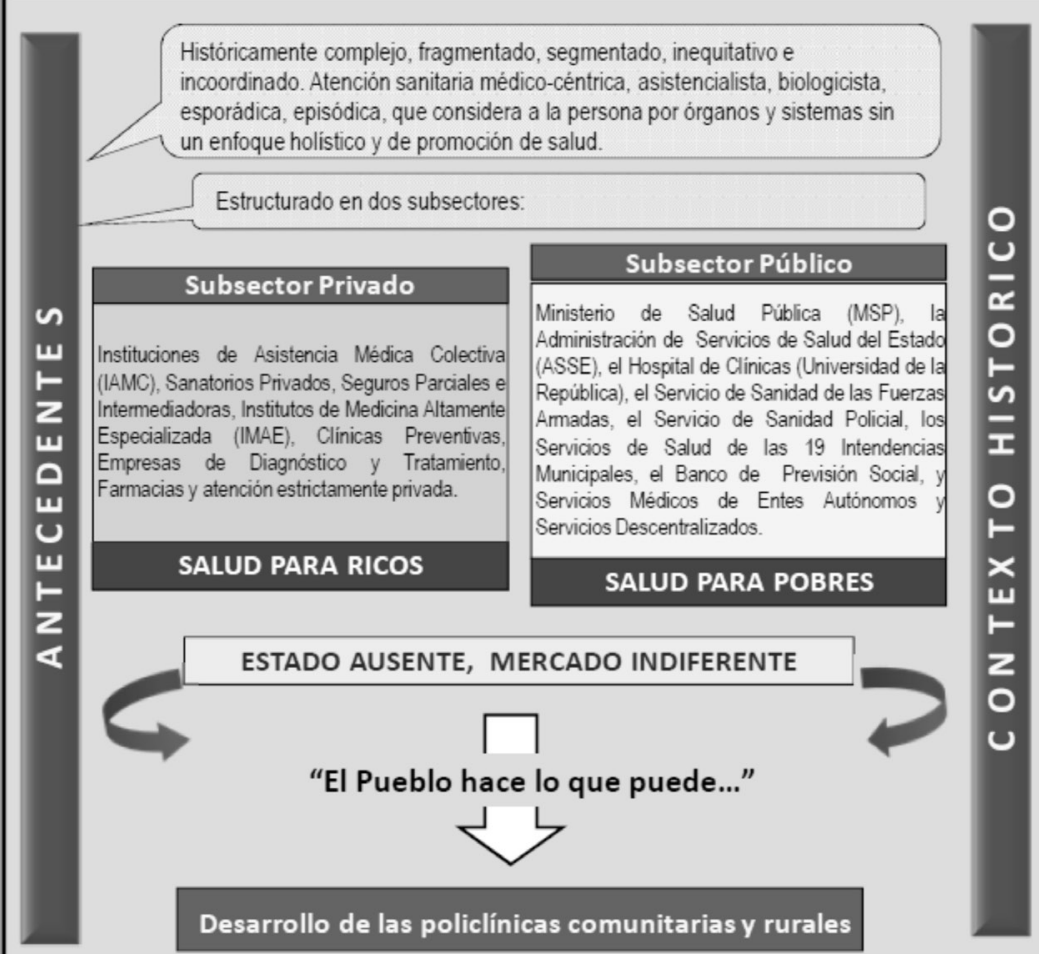

\section{LA LUCHA POR EL DERECHO A LA SALUD}

En Uruguay la lucha por el derecho la Salud, parte de multiples luchas sociales, desde sindicatos, aun durante la dictadura, resistiendo el desmantelamiento de lo publico a mano del neoliberalismo de los 90

Esta lucha es retomada en los primeros Foros Sociales (20022004) y Foros Sociales de la Salud (2005-2008), en los que participaron organizaciones de la sociedad civil que reivindicaban el derecho a la misma

- En 2005 se da el giro politico hacia la izquierda con la Presidencia del Dr. Tabaré Vazquez liderando el Encuentro Progresista-Frente Amplio-Nueva Mayoría (hoy Frente Amplio) en el gobierno nacional.

Se inicia un cambio en la concepción de país, de sociedad y del "hombre", respaldado por más del $50 \%$ de la ciudadanía.

El pais que recibió el gobierno del Frente Amplio, podria caracterizarse en:

Deuda externa,

Deuda social,

Desmantelamiento productivo

Indulto a los violadores de derechos humanos.

- El Cambio es ratificado en 2009 con la elección de José Mujica como Presidente de la República.

\section{Gráfico 2. Sistema de salud en Uruguay (2)}

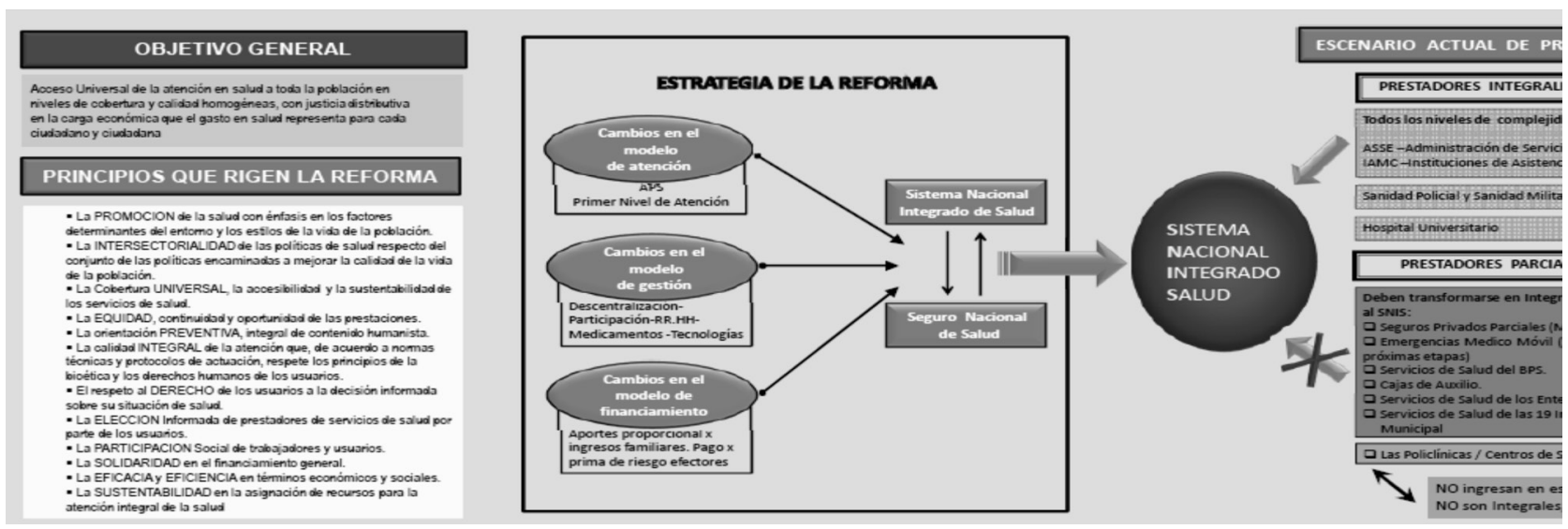


coberturas parciales, comúnmente denominados 'seguros parciales', que buscaron 'captar' a ese nicho de mercado intermedio, 'los que podían pagar, pero menos'. Para ello, desarrollaron en su mayoría seguros quirúrgicos parciales - por lo que, muchas veces, las personas se encontraban con que lo que había contratado no cubría su necesidad asistencial -. Bajo esa misma modalidad se desarrollaron los servicios de 'Emergencia Médico Móvil', los seguros y servicios odontológicos colectivos/prepagos, y en menor medida, los de atención psicológica. Existen también otros prestadores parciales o integrales no incorporados aún al SNIS: servicios de salud públicos que sólo desarrollaron el primer nivel de atención (policlínicas municipales); otros que desarrollaron áreas de atención especializadas como el Banco de Previsión Social (BPS); e incluso el Hospital de Clínicas de la Universidad de la República (UdelaR), que es un prestador integral aunque con escaso desarrollo del primer nivel respecto a las áreas de 3er y 4to nivel de complejidad; finalmente, otros servicios de salud universitarios que realizan algunas prestaciones asistenciales y acciones de promoción de salud comunitaria.

En la situación actual se visualizan avances (BORGIA, 2009) pero "los problemas de inequidad de la salud en Uruguay aún no se han resuelto" (BORGIA, 2008, p.142). Sin embargo, es una idea errónea pensar desde que los problemas de salud se resolverían con una reforma del sector, hasta que toda la evidencia de la epidemiología crítica y la medicina social/salud colectiva latinoamericana (ALMEIDA-FILHO, 1992; ALMEIDA-FILHO, 2000; AROUCA, 2008; BREILH, 2003; BREILH, 2010; GARCÍA, 1991; GARCÍA, 1994; GRANDA, 2009; LAURELL-NORIEGA, 1989; LAURELL, 1997; ROJAS-MÁRQUEZ, 2009; SAMAJA, 1993) y recientemente de la OMS (2008; 2011), indican que la vía para garantizar el derecho a la salud, además de sistemas de salud universales, multiculturales e inclusivos, implica generar políticas públicas que actúen de manera contundente sobre el proceso de determinación social de la salud (ALAMES; CEBES, 2011; CEBES, 2009; NOGUEIRA, 2010) y sobre los determinantes sociales de la salud que se interrelacionan y condicionan entre sí y con el conjunto de los derechos humanos (BORGIA, 2005).
La reforma del sistema está en proceso en su implementación y en su concepción. La manera en que se lleva a cabo la disputa de sentido: 'garantía del derecho a la salud con fuerte participación social', o por el contrario, más 'mercado protegido/subsidiado, medicalización y tecnificación', determinarán el futuro de la misma y de la salud en Uruguay. La participación autónoma y comprometida de usuarios y trabajadores en la dirección de las políticas sanitarias, tanto en la Junta Nacional de Salud como en ASSE, será clave en este sentido, siendo un punto de inflexión su incorporación a la tarea de "co-conducción del sistema y su reforma" (BORGIA, 2008, p.142).

\section{Justificación de la Investigación}

Si bien la reforma se orienta hacia la consolidación de prestadores integrales de salud, en 2008 persistía aún la indefinición sobre la relación de las policlínicas de origen comunitario y/o rural con el SNIS. En estas últimas, por la vía de los hechos y debido a su lejanía de los centros asistenciales, la cobertura quedaba en 'terreno de nadie' ante la demanda expectante de la población en zona rural, no existiendo en el momento del comienzo de la investigación mensajes claros por parte del Gobierno en cuanto a cuál sería el lugar de estas policlínicas comunitarias y frente al interés creciente por parte de los prestadores privados en captar usuarios (que ahora capitanean y por tanto son rentables). Además, es importante mencionar que este proyecto de investigación surge en el marco del Programa de investigación Teasdale-Corti Revitalizando la salud para todos, cuyo alcance fue ampliado durante la reunión internacional de discusión metodológica (Bogotá, 2008), y en el proceso participativo de su ejecución.

\section{La Investigación}

El estudio tuvo como Objetivo General rescatar la historia y el contexto de la conformación de las Policlínicas Comunitarias y generar con la comunidad las propuestas para el desarrollo de la APS-I y su integración al 
SNIS, llevando a cabo acciones políticas que transformaran las propuestas en parte de la política sanitaria del SNIS. Los objetivos específicos fueron: 1) recuperar la memoria de las policlínicas rurales y comunitarias urbanas; 2) analizar las maneras de entender la APS y formular propuestas para el desarrollo de la APS-I en las policlínicas comunitarias; 3) analizar el proceso de conformación del SNIS y formular propuestas para la integración de las policlínicas comunitarias; 4) acordar y llevar a cabo un plan de acción para la concreción de las propuestas; 5) socializar reflexiones y aprendizajes, fomentar el intercambio con equipos de investigación y organizaciones comunitarias de Latinoamérica y el mundo.

\section{Metodología}

Se utilizaron: a) investigación-acción-participativa (IAP), b) métodos cuantitativos de análisis de datos y c) la revisión bibliográfica y documental.

a) El abordaje de IAP aplicado aquí se refiere a la concepción latinoamericana basada en la teoría antropológica (función de los universos simbólicos en el mantenimiento del statu quo), y desde la sociología crítica de origen marxista (se explica el orden establecido por razones económicas), situaciones que para ser cambiadas requieren la toma de conciencia por parte de aquellos que pertenecen a la clase explotada y dominada (ANDER-EGG, 2003). Fals Borda comienza a partir de 1962 a cuestionar el positivismo sociológico desarrollando una conciencia social transformadora orientada hacia el compromiso revolucionario (GUERRERO; GARCÍA; SÁNCHEZ, 2011). En este contexto, trabaja sobre el concepto de ciencia y conocimiento, que debe estar al servicio del cambio y contribuir a la emancipación de los sujetos investigados (FALS BORDA; RAHMAN, 1991). Apoyándose en Kuhn (2005), cuestiona las bases epistemológicas de la producción de conocimiento, y propone una transformación en los propios investigadores que les permita una nueva forma de ver los procesos populares, o lo que él llamó 'un ver de nuevo'. En esta construcción del saber desde la mirada del oprimido y del explotado es donde se pueden producir las transformaciones a nivel de las estructuras sociales. Por su parte, Rodrigues Brandao (CETRULO, 1987) no comparte que la investigación participativa constituya un cambio de paradigma en la producción de conocimiento. El que sea participativa marca un compromiso social del científico, el cual no dependería de la teoría con la cual se acerque a la realidad. Propone democratizar el saber que se produce en la Universidad, comprometiéndolo con la causa popular, usándolo en un proyecto que se encuentro bajo 'poder popular'; considera que el saber académico es tan necesario e importante como el saber popular, con el cual aquel debe articularse pero no perderse, por lo que propone la investigación participativa, en el sentido de 'investigación comprometida' (CETRULO, 1987). En este mismo contexto revolucionario de los años 70, otro abrevadero de la IAP fue el movimiento de Educación Popular iniciado en Brasil con el pedagogo Paulo Freire, que educando a los más pobres de entre los pobres desarrolló la pedagogía para la liberación de los oprimidos, trabajando específicamente desde el plano ideológico en lo que llamó 'la concientización' que los llevaría a tomar conciencia de clase y luchar contra la opresión. El saber académico y el saber popular debían respetarse mutuamente y nutrirse el uno al otro en aras de la liberación (FREIRE, 1993).

A partir de estas raíces, un vasto número de investigadores comprometidos con las reivindicaciones en torno a la justicia e igualdad ha desarrollado una forma de contribuir al camino de la 'liberación de la explotación económica y de la alienación ideológica y cultural' desde la reflexión de los propios implicados sobre su vida cotidiana, su cultura y saber empírico y muchas veces mágico, proponiendo categorías cuestionadoras, de tal manera que 'lo obvio fuera releído'. Mediante la fuente de la historia latinoamericana y el potente desarrollo metodológico de la IAP y 'su utilización en esta investigación', se pretendió desestabilizar la supremacía del gobierno y los actores privados en el acceso a la información; propiciar espacios de reflexión y análisis en un plano de horizontalidad que habilitara a la comunidad a realizar propuestas; trabajar con una concepción de la realidad como hecho construido y por tanto que pudiera ser cambiado; generar propuestas 
sólidas que respaldaran y legitimaran acciones políticas para transformar las propuestas comunitarias en política sanitaria.

El proceso de investigación implicó una secuencia continua de trabajo en y con la comunidad, la aplicación de cuestionarios mediante entrevista a personal y referentes comunitarios vinculados a cada una de las policlínicas, la sistematización, reflexión, síntesis de la información relevada y la puesta en común colectiva en foros comunitarios (véase el gráfico 3). Complementariamente se realizaron las siguientes actividades y acciones:

a1) Encuentros de Policlínicas Rurales y Comunitarias, se propiciaron encuentros entre autoridades sanitarias nacionales y pobladores/usuarios de diferentes localidades, instancias en una de las cuales las autoridades recibieron en mano un documento de propuestas a ser incluidas en el próximo presupuesto nacional; en el proceso surgió la inquietud por presentar proyectos de promoción de salud y realizar cursos de capacitación.

a2) Proyectos de promoción de salud (dos) en el marco de la convocatoria a concurso del Programa Uruguay Saludable del MSP, como acciones concretas por la salud de las comunidades, construidas participativamente con apoyo técnico del equipo de investigación e incorporando otros técnicos y profesionales al proceso. Actualmente uno de los proyectos está finalizando la ejecución (en el departamento de Salto) y el otro iniciándola (en el departamento de Soriano).

a3) Cursos de capacitación, en los meses de agosto y setiembre de 2009 se coordinó con el Movimiento Nacional de Usuarios de Servicios de Salud Pública y Privada (MNUSPP) una propuesta de capacitación que contó con docentes y facilitadores/as de ALAMES, OPS/OMS, MSP y la Intendencia de Montevideo, que además brindaron apoyo económico, y la Administración Nacional de Educación Pública (ANEP) que brindó apoyo locativo. Se capacitaron a unas 100 personas, organizadas en dos grupos, provenientes de todo el país, varias de pequeńas localidades del área rural. El programa exigió una dedicación horaria de unas 50 horas, de las cuales: 14 horas eran de trabajo domiciliario, y una modalidad de trabajo presencial intensiva durante dos fines de semana completos para el primer grupo y de un fin de semana extendido para el segundo grupo. La propuesta genérica de contenidos se estructuró sobre cuatro ejes de trabajo: a) abordajes diversos de conceptos básicos; b) políticas y sistemas de salud; c) salud y reforma en Uruguay; d) promoción de salud y programas sanitarios nacionales. Los debates e intercambios de realidades y experiencias dieron lugar a situaciones recurrentes de denuncia pública de salud rural y de salud mental, proponiéndose hacer replanteamientos en los ámbitos de derechos humanos en lo nacional, regional e internacional. Se realizaron denuncias ante el MSP, en la prensa local y nacional e intervino la justicia (en lo referente a las 'colonias psiquiátricas').

a4) Los aspectos centrales de las situaciones planteadas en el punto anterior fueron incorporados al Informe Alternativo sobre los Derechos Económicos, Sociales y Culturales en Uruguay, y tomados en las Recomendaciones del Comité de Expertos de la Organización de las Naciones Unidas (ONU). El informe alternativo fue elaborado por el Capítulo Uruguay de la Plataforma Interamericana de Derechos Humanos, Democracia y Desarrollo. Respecto del Artículo 12º: Derecho a la salud, el informe dice lo siguiente: "Reconocemos que la reforma sanitaria que se está realizando en el país es un avance respecto al ejercicio y las garantías del derecho a la salud. Sin embargo, la universalización de esta reforma no es igual en todo el país. Los datos muestran que a medida que aumenta la distancia desde la capital del país menor es el grado de integralidad de las políticas. La centralidad de la capital departamental y de las ciudades medianas genera diferencias con los pueblos y comunidades rurales. En la mayoría de las zonas rurales no hay profesionales de la salud radicados, contándose solamente con auxiliar de enfermería o promotora rural y una ronda médica que visita la localidad con una frecuencia que va de 15 días a tres meses. Esto genera serias dificultades de acceso porque las vías rurales están en malas condiciones. Esto se agudiza con la carencia de transporte regular de pasajeros". "Las expertas del CEDAW manifestaron en 2008 su preocupación por 


\section{(...) el hecho de que no haya ninguna institu-} ción pública ni ninguna politica que se ocupe de las mujeres de las zonas rurales, en particular en lo que respecta al empleo, la salud y la educación. (p. 42).

En consecuencia, recomiendan al Estado Uruguayo que "(...) elabore políticas y programas integrales destinados a mejorar la situación económica de las mujeres de las zonas rurales, facilitándoles el acceso a la capacitación, los recursos de producción y el capital, así como a los servicios de salud y la seguridad social (p 43)". "Recomendación: Que el Estado Uruguayo implemente políticas públicas de salud hacia la población en zonas rurales garantizando el derecho a la igualdad de oportunidades y derechos" (CAPÍTULO URUGUAY-PIDHDD, 2010, p. 21). El Comité de Expertos de la ONU recomienda

al Estado que en su próximo informe periódico facilite información estadistica actualizada, incluidos datos, sobre la aplicación efectiva de cada uno de los derechos recogidos en el Pacto, desglosada por edad, sexo, población urbana o rural, situación socioeconómica(...); la reubicación en viviendas seguras de las familias que se encuentran en asentamientos irregulares, la prestación de asistencia a las familias con bajos ingresos y otras personas y grupos desfavorecidos y marginados, y el suministro de servicios de saneamiento adecuados; (...) además de seguir intensificando la aplicación de medidas para asegurar el acceso a una atención de la salud universal, entre otros medios, paliando los desequilibrios regionales en el acceso a dicha atención y haciendo frente a la incidencia de enfermedades prevenibles en los niños". "También alienta al Estado a que siga recabando la participación de las organizaciones no gubernamentales y otros miembros de la sociedad civil en el proceso de debate a nivel nacional (...). (CAPÍTULO URUGUAY-PIDHDD, 2010, p. 30, 35-38). a5) Foros Departamentales-Regionales de Salud, se apoyó en la experiencia de autoconvocatoria de los tres Foros Sociales de Uruguay (2002-2004), los cuatro Foros Sociales Uruguayos de Salud (2005-2008), el Primer Foro Social Sudamericano de Salud (2007), de los seis Foros Sociales Regionales de Salud (2007), constituyendo un accionamiento relevante en la articulación entre organizaciones sociales y personas preocupadas por la salud en el país. La potencialidad de la misma parte de la posibilidad de constituir un espacio regular de encuentro, intercambio, debate, enseñanza/aprendizaje y coordinación nacional, vinculada con las Asambleas Anuales del MNUSPP (2005-2008 y 2010), y enriquecida por la vinculación con procesos similares en la región y el mundo. Esta experiencia ha sido desarrollada sin desconocer que en el mismo período se han desarrollado otros esfuerzos de articulación como el proceso del II Congreso del Pueblo y que se han promovido nuevos ámbitos institucionales para la participación social organizada como los Consejos Sociales, los Encuentros de Mujeres, etc. Pese a lo precario de la capacidad organizativa y de convocatoria del equipo de investigación y de las organizaciones aliadas, los mismos han logrado involucrar, consultar, participar y acercar ideas de un número inusual de personas (900), y más aún de personas del Uruguay profundo (más de 50 localidades de todo el país, mayoritariamente rurales). Esto sólo ha sido posible porque ha habido colectivos de gente preocupada y con capacidad organizativa en diversos lugares, porque han logrado obtener el apoyo de pequeños comercios y autoridades locales, y porque el MNUSPP contó con el apoyo del MSP (aunque se pretendió un apoyo mayor). Obviamente, las actividades no habrían sido posibles solamente con los recursos del proyecto de investigación, sin contar con estos múltiples apoyos y compromisos. La necesidad de síntesis para la acción requirió conectar experiencias diferentes entre comunidades que no se conocían entre sí, a fin de generar un relato-síntesis compartido y socializado en plenario de representantes de las localidades (en una primera instancia). En ese sentido, es evidente que los tiempos del proyecto no permitieron agotar los procesos de reflexión comunitaria, sino que apenas la provocaron con mayor o menor grado de avance según las regiones. 
b) Métodos Cuantitativos: b1) el análisis de 'los uruguayos/as y la salud: situación, percepciones y comportamientos" está basado en el procesamiento de los microdatos de la Encuesta Continua de Hogares Ampliada 2006 (ECHA 2006) del Instituto Nacional de Estadísticas (INE). El tamaño de la muestra anual es de 87.228 viviendas y 256.867 personas distribuidas en un 35\% en Montevideo; $3 \%$ en la periferia metropolitana (pequeñas localidades ubicadas hasta $30 \mathrm{~km}$ del centro de Montevideo); el 31\% corresponde al Interior urbano residente en localidades de 5.000 habitantes o más; el 12 $\%$ corresponde a localidades de menos de 5.000 habitantes; y el $19 \%$ a población rural dispersa (en adelante nos referiremos a población rural como aquella que vive en localidades de menos de 5.000 habitantes y en áreas rurales propiamente dichas). Como la ECHA 2006 incluye módulos temáticos específicos que se indagan por trimestre, los datos referidos al módulo de salud fueron relevados en el tercer trimestre e incluyen a 64.164 personas.

Para analizar los 'cambios en la cobertura sanitaria de la población del país', se utilizaron los microdatos de la ECHA 2006 y las serie de microdatos de las ECH del período 2007-2009 (última disponible entonces). El tamaño total de la muestra es de 657.103 casos correspondiendo el $39,1 \%$ al año 2006, el 18,7\% al año 2007, el 22\% al año 2008 y el 20,2\% al año 2009.

b2)La población rural de acuerdo al Censo 2004 Fase I (INE) en localidades de menos de 5.000 habitantes era de 323.750 personas, mientras que en el área rural dispersa era de $266.289,10,5 \%$ y $8,2 \%$ respectivamente, evidenciándose una tendencia al despoblamiento progresivo. Para el período 2008 a 2010, se recurrió a las estimaciones de Paulino y Pereda (2008) que proyectaban un $8,9 \%$, dado que la misma se ubica en un rango intermedio entre los hallazgos del Censo 2004 Fase I y las estimaciones de 6,9\% de Riella y Mascheroni (2009), y se basan en las realizadas por el INE.

b3)Para el cálculo de los recursos financieros, a partir de las ECH 2008 y ECH 2009 se ha calculado la población rural, por sexo y edad que tenía cobertura de salud a través del FONASA, siendo ésta un 40,3\% y un $48,5 \%$ del total respectivamente. Dichos cálculos se multiplican por el valor diferencial de las cápitas (OLESKER, 2008; PILÓN, 2009), por el peso relativo de cada grupo de población (sexo, edad, año) y por el porcentaje de población rural cubierta por el FONASA cada año. En segundo término, se estiman los egresos por concepto de metas, tickets y FNR (JUNASA, 2008; JUNASA 2009), realizando un prorrateo de las transferencias sobre la base de los egresos por cápitas calculadas previamente para la población rural. Por último, se convierten los valores en pesos corrientes al valor dólar promedio anual comprador, se suman los importes de los años 2008 al 2010, para luego prorratear por departamento y tipo de área rural. Los egresos del año 2010 se estimaron como semejantes en U\$S a los del año 2009, dado que la leve merma de población tendencialmente se compensa con el aumento de la población con cobertura FONASA, de los valores de la cuota FONASA y con un dólar estable (operando por momentos con tendencia a la baja).

Esta proyección lleva a una discusión de fondo: ¿los recursos permitirían llevar adelante una atención en el primer nivel con condiciones de calidad? Para poder responder a esa pregunta, es necesario hacer dos cálculos complementarios: i) el costo de una nueva y equipada Policlínica Rural que permita realizar una atención de las tres especialidades básicas (medicina general, pediatría y ginecología) siguiendo los parámetros establecidos en la normativa para la habilitación de servicios de salud, que se estimó en U\$S 100.000, incluyendo salón multiuso y apartamento médico.

ii) el costo de las horas médicas para la atención diaria, de lunes a sábados, 4 horas por cada una de las tres especialidades médicas básicas. Como no existe un único valor hora, se tomó la cifra de la salud privada (SMU, 2010) por ser el mayor valor (sueldo base medicina general por policlínica de 26 horas mensuales), y se estimó para el pago por acto médico (co-pago por paciente atendido) una consulta de 4 pacientes por hora en una consulta de 4 horas diarias (16 pacientes).

b4) Para indagar acerca de 'la situación de las policlínicas comunitarias y/o rurales de Uruguay' un primer aspecto era: ¿qué se entiende por policlínica, por consultorio y por puesto o posta de salud? Dado que muchas veces se utilizaban indistintamente estos conceptos entre las personas consultadas, se realizó la búsqueda de servicios de salud ambulatorios. En términos operativos se entendió como 'comunitarias': 'aquellas que tienen o tuvieron 
alguna forma de participación (en sentido laxo) de la comunidad' (el préstamo o cesión del predio/construcción, la operativa cotidiana, etc.); y como 'rurales' 'aquellas ubicadas en localidades de menos de 5.000 habitantes o en zona rural propiamente dicha' (INE). Por tanto, en este estudio se incluyen de manera no exhaustiva servicios de salud rurales de diferentes orígenes y características (sean estos comunitarios o no), y de entre los urbanos, aquellos que son comunitarios.

b5) Para valorar en qué medida dichos servicios cumplían con los requerimientos para las policlínicas que establece el Decreto 416/2002: "Policlínica: es el conjunto de varios consultorios instalados en una misma planta física que están vinculados entre sí, con una actividad en equipo, y que deberá contar con una Dirección Técnica responsable. Deberá brindar atención médica al menos en tres de las Especialidades Básicas: Medicina General, Pediatría y Ginecología (...) Contará además con servicios intermedios de apoyo (enfermería, farmacia interna, etc.)". "Los servicios deberán contar con un Sector Administrativo en el que funcionará el Archivo"; el equipamiento debe ser adecuado para la función, estar en perfecto estado de higiene, conservación y funcionamiento, además de contar con un servicio técnico responsable de su mantenimiento; el personal técnico debe estar habilitado y registrado en el MSP. A efectos del análisis se consideró a los efectores del primer nivel de atención incluidos en el estudio, respecto de las características que deberían reunir para ser considerados o tener un funcionamiento acorde a una policlínica (véase gráfico 3).

b6) Se indagó sobre 'la accesibilidad y las disposiciones normativas', para lo que se partió del considerado en el Decreto 359/2007, que sostiene que: "el tiempo de espera constituye un elemento a tener en cuenta a la hora de evaluar la accesibilidad de los usuarios a las prestaciones"; "que asimismo permite relacionar la oferta de Servicios con la demanda asistencial, a partir de la disponibilidad de los recursos humanos destinados al área"; "que también constituye un indicador sensible, que permite monitorear la calidad de la asistencia que se ofrece a los usuarios". Tomando en cuenta las
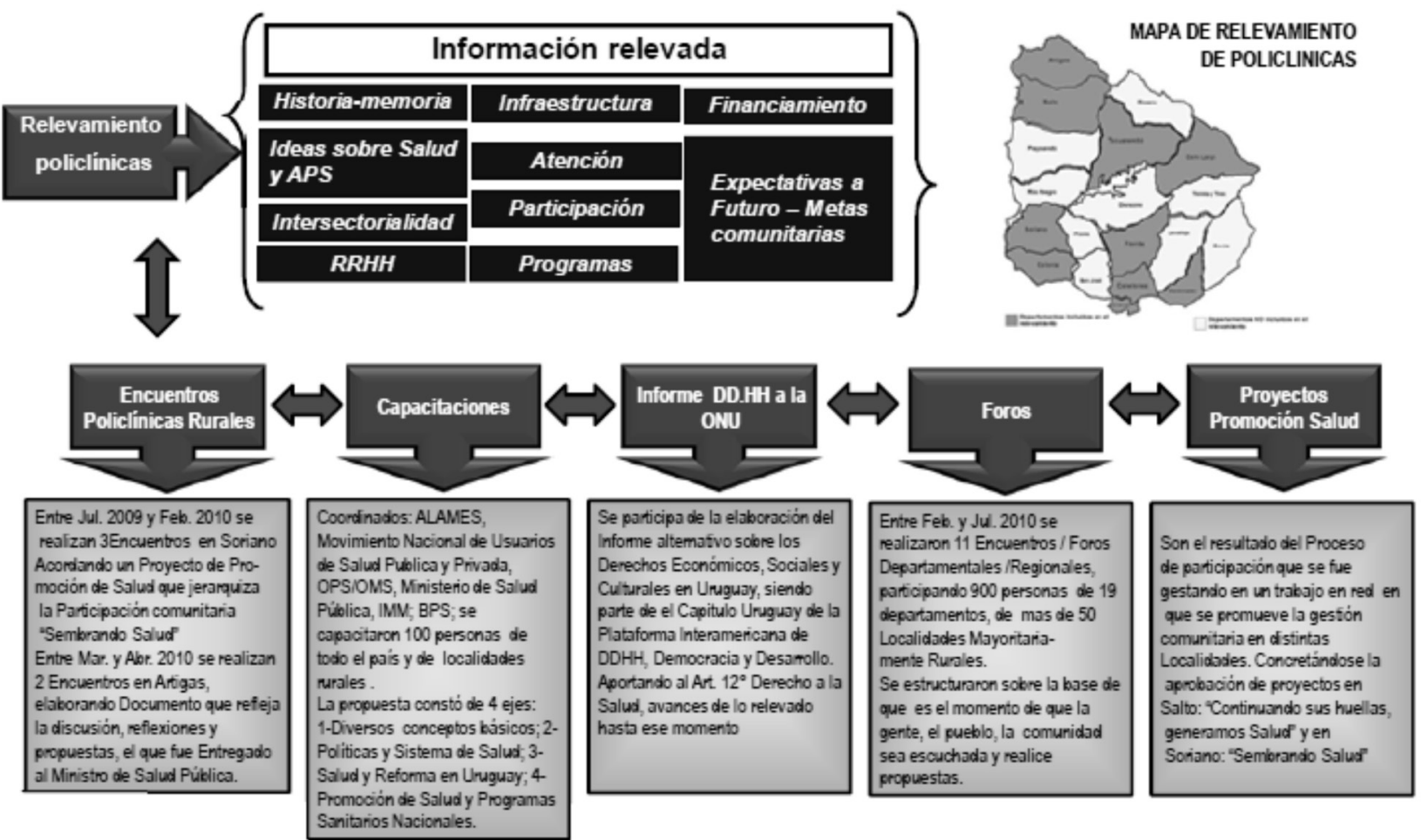
consideraciones precedentes, se indagó sobre los tiempos de espera y la disponibilidad de recursos humanos como mecanismo de evaluación de la accesibilidad real de la población a los servicios de salud. El decreto n.464/2008 reglamenta los artículos 15 y 28 de la Ley 18.211, estableciendo las cláusulas de los "contratos de gestión" que la Junta Nacional de Salud (JUNASA) suscribirá con los prestadores que integran el SNIS. A efectos de este análisis, resulta relevante recordar lo establecido en dos cláusulas de dichos contratos de gestión: N.35 - Tiempos de espera.

El prestador se obliga a dar cumplimiento a lo dispuesto en el Decreto 359/007 del 28 de setiembre de 2007 y a las demás normas referidas a tiempos de espera que establezca el Ministerio de Salud Pública";

N.36 - Horarios de atención del primer nivel.

El horario de disponibilidad de la consulta de medicina general, pediatría, de los servicios de apoyo administrativos y para la realización de exámenes paraclínicos, será de 8.00 a 20.00 horas, de lunes a viernes y de 8.00 a 14.00 horas en dias sábado. Estas obligaciones serán exigibles en la sede principal, promoviéndose su extensión a las sedes secundarias o filiales del prestador, que podrán cumplirlas mediante acuerdos de complementación con base territorial con otros prestadores públicos o privados.

La normativa diferencia geográficamente, exigiendo ciertos días y horarios para las sedes principales, y promoviendo su extensión a las sedes secundarias o filiales. A efectos del análisis se evaluó a los servicios ambulatorios del primer nivel de atención estudiados respecto a las obligaciones de tiempos de espera y horarios de funcionamiento que se exigen en la sede principal y se promueven en las sedes secundarias (véase gráfico 3).

b7) Alcance y limitaciones del relevamiento: fue realizado entre marzo de 2009 y enero de 2011, es de 119 policlínicas, de 10 (en un total de 19) departamentos (estados, provincias): Artigas, Canelones,
Cerro Largo, Colonia, Florida, Maldonado, Montevideo, Salto, Soriano y Tacuarembó, siendo $70 \%$ rurales y el 30\% comunitarias/urbanas (véase gráfico 3); el estudio incluye la totalidad de las policlínicas rurales de Salto (38), de las rurales y comunitarias de Artigas (27) y Cerro Largo (22). Si bien el tamaño de la muestra podría ser representativo del país, la metodología de selección de casos no buscaba una validez estadística, y en consecuencia los datos agregados deben tomarse como tendencias problematizadoras del funcionamiento del sistema de salud respecto de este tipo de prestadores y no del primer nivel de atención en su conjunto (véase gráfico 3).

c) Revisión bibliográfica y documental: la misma, por razones del espacio asignado a la publicación de este artículo, se incluye parcialmente en la bibliografía.

\section{Principales Conclusiones}

El propósito principal de la investigación era el de poner en la agenda sanitaria la necesidad de una política nacional de salud rural y una estrategia de incorporación de las policlínicas comunitarias y rurales al SNIS (véase grafico 5). Evidentemente, se buscó que esto ocurriera respetando los procesos sociales-comunitarios, los saberes populares-rurales y sin recetas tecnocráticas de escritorio. Desde este punto de vista, se puede afirmar que el principal objetivo, poner en la agenda a la salud rural, se ha cumplido. Podrían ensayarse múltiples explicaciones del por qué esto ha ocurrido y, seguramente, en varias de ellas esta investigación tendría poco que ver. Si bien hay varias organizaciones rurales y comunitarias que hace tiempo vienen luchando por soluciones para la población rural, hay algunos elementos que hacen suponer que la tarea resultó exitosa producto de la acumulación de estos múltiples esfuerzos previos y contemporáneos,: a) en el programa del FA (FRENTE AMPLIO, 2008; FRENTE AMPLIO 2009; ASTORI, 2009) no había ninguna mención al tema de la salud rural durante la extensa campaña electoral, ningún partido político o medio de comunicación masiva hizo mención al tema; b) se tuvieron tres reuniones previas (a la asunción) con las autoridades sanitarias entrantes (del gobierno electo 
en 2009 y que asumió el 1 ro de marzo de 2010), en las que se les anunció la tendencia central de los resultados; c) el primer pronunciamiento público sobre la formulación de un programa de salud rural y su priorización por parte del gobierno en el período (2010-2014) fue en el 26 de febrero de 2010 en el marco del Segundo Encuentro del Proyecto "Revitalizando la Salud para Todos: Aprendiendo de la Atención Primaria en Salud Integral" Montevideo-Maldonado, Uruguay; d) el $1^{\circ}$ de marzo de 2010 el Ministro de Salud hace referencia a la salud rural como prioridad; e) en las presentaciones públicas de los Encuentros-Foros de Maldonado, Tacuarembó, San José y Paysandú se verificó que no había una propuesta concreta del programa de salud rural, sino que la idea fue madurando en la formulación de una metodología de construcción de la propuesta, con participación de los equipos de salud en los territorios; f) el equipo de dirección-coordinación de salud rural del Ministerio acuerda en el Foro-Encuentro de Paysandú la consulta al MNUSPP que concurre a varios talleres y acuerda designar un referente para el tema en cada departamento.

La historia de la génesis de muchos de los servicios de salud rurales y comunitarios está en riesgo de caer en la desmemoria. Indagar sobre estos aspectos genera 'inquietud' en las personas entrevistadas, en algunos la historia es narrada con notorio sentimiento de pertenencia y orgullo como por ejemplo: Policlínica UTAA en Bella Unión o Policlínica Solidaridad en el barrio Flor de Maroñas de Montevideo; en otros la persona idónea sea ha ido y con ella gran parte de la memoria (que habría que recuperar) de luchas y esperanzas. Es necesario rescatar los saberes populares, no borrar huellas de luchas pasadas, promover a la comunidad como sujetos de derechos, y fundamentalmente respetar sus tiempos para apropiarse de lo nuevo adaptándolo a su sistema de vida. La cuestión de la historia local y su policlínica no es destacada en el discurso de la autoridad sanitaria, donde prevalece la idea de racionalidad del gasto y que aquello que pudo haber sido bueno o necesario en su momento, ahora quizás no lo sea tanto. Pese a ello, en un contexto de múltiple recurrencia discursiva a una crisis de la participación social, las policlínicas logran mantener un cierto dinamismo de participación, y aquellas experiencias que surgieron verticalmente, posteriormente requirieron de un soporte horizontalcomunitaria para sobrevivir (véase gráfico 4).

La accesibilidad (véase gráfico 5). En las expectativas recogidas en la comunidad tales como: "que asista un pediatra una vez por lo menos cada tres meses" y "especialistas como ginecólogo, cardiólogo aunque sea dos veces al año" impactan las ausencias, evidenciándose el desconocimiento de derechos fundamentales y del derecho a la salud en particular. Impacta la brecha entre lo esperado y la normativa vigente. En estas poblaciones rurales y periféricas no se ha concretado aún la apropiación de un concepto integral de salud que parta de la promoción y el cuidado. Las experiencias de contacto con el derecho a la salud han estado relacionadas con la enfermedad: 'se atiende', 'se asiste', 'se alivia' y se relaciona principalmente con la atención médica. Esta relación con los servicios de salud hacen del usuario un ser que 'es pasivo', 'que padece', no pudiendo asumirse como sujeto activo que ejerce derechos, denuncia, propone, cuestiona y aporta. Hablar de APS-Integral en estas situaciones es hablar de algo desconocido, nuevo para los entrevistados, generando dudas y nuevas preguntas. En este contexto se palpa la significación de la dimensión relacional de la accesibilidad que afecta el ejercicio del derecho a salud, entendida como

\section{(...) un vinculo que se construye entre los usu- arios y los servicios. Este vinculo surge de una combinatoria entre condiciones y discursos de los servicios y las condiciones y representaciones de los sujetos, y se manifiesta en la modalidad particular que adquiere la utilización de los servicios". (STOLKINER, 2000, p. 282).}

La accesibilidad cultural o simbólica se expresa en diferentes imaginarios, lenguajes y concepciones en relación a la salud enfermedad/atención; puede propiciar o impedir el encuentro entre los servicios y la población potencialmente usuaria, así como utilizarse para justificar la no garantía de derechos. Cuando gran parte de la población rural se integra a las IAMCs como resultado de la intensa campaña de afiliaciones desarrolladas en localidades rurales y periferias urbanas por parte de los 


\begin{tabular}{|c|}
\hline $\begin{array}{l}\text { edictadura y dos tercios de las mismas en post dictadura. } \\
\text { egularmente } 90 \% \text { ante situaciones puntuales. } \\
\text { le las policlínicas fundadas por vecinos que formaron comisiores } \\
\text { tegradas por los mismos, tres cuartas partes continúan organizadas de la } \\
\text { isma forma, mientras que el } 15 \% \text { incluyó o delego en terceros la gestión. } \\
\text { le las policlínicas fundadas por vecinos conjuntamente con otros y que } \\
\text { maron comisiones, el } 100 \% \text { de las que continúan, lo hacen sólo con la } \\
\text { raticipación de la comunidad. } \\
\text { las polusivamente gracias a la participación comunitaria. }\end{array}$ \\
\hline 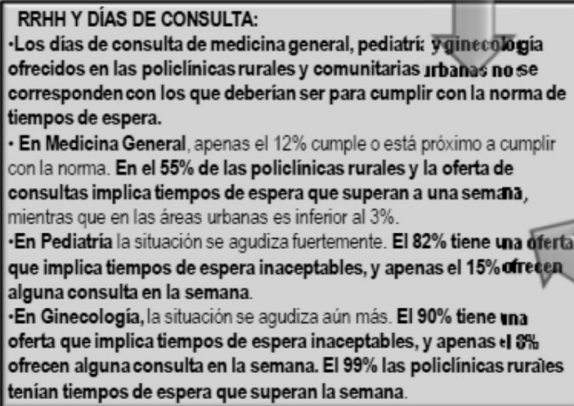 \\
\hline
\end{tabular}

\section{ORGANIZACIÓN Y PARTICIPACIÓN EN LAS POLICLINICAS:} - De las polinicas incorporadas al estudio, la cuarta parte fue und - Casi el 90\% de la co para el trabajo colectivo el $25 \%$ se reuine con regularidad yel 5 ) $\%$ irregularmente 0 ante situaciones puntuales.

- De las policlinicas fundadas por vecinos que formaron comisiores integradas por los mismos, tres cuartas partes continúan organizadas de la - De las policlíicas fundadas por vecinos conjuntamente con otros y que participación de la comunidad.

- Los dias de consulta de medicinageneral pediatri y yineconog ofrecidos en las policlínicas rurales y comunitarias intanas nose tiempos de espera.

En Medicina General, apenas el $12 \%$ cump e esta proximo a cun mientra que enlas áres unanas es inferior $13 \%$.

-En Pediatría la situación se agudiza fuertemente. El $82 \%$ tiene una dfert que implica tiempos de espera

ofrecen algunaconsulta en la semana El $99 \%$ las policlínicas ruraies tenian tiempos de espera que superan la semana

\begin{tabular}{|c|c|c|c|}
\hline \multicolumn{4}{|c|}{ 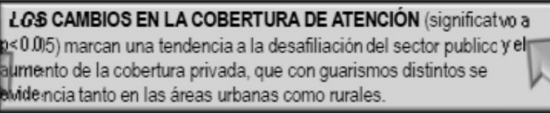 } \\
\hline \multirow{2}{*}{\multicolumn{2}{|c|}{ PRINCIPALES TIPOS DE COBERTURA SANITARIA }} & \multicolumn{2}{|c|}{ AR̃O DE LA ENCLESTA } \\
\hline & & 2006 & 2009 \\
\hline \multirow[t]{4}{*}{ MONTEVIDEO } & $\begin{array}{l}\text { COBERTURA PÚBLLCA (MSPIASSE y } \\
\text { HOSPITAL DE CLLINCAS) } \\
\end{array}$ & $37,7 \%$ & $21,9 \%$ \\
\hline & $\begin{array}{l}\text { COBERTURA PRIVADA (IAMC y } \\
\text { Seguros Privados Integralas) }\end{array}$ & 49,05 & $68,0 \%$ \\
\hline & OTROS & 13,35 & 10,15 \\
\hline & TOTAL & $100,0 \%$ & $100,0 \%$ \\
\hline \multirow[t]{4}{*}{$\begin{array}{l}\text { INTERIOR } 5000 \\
\text { YMAS }\end{array}$} & $\begin{array}{l}\text { COBERTURA PÚBLICA (MSPIASSE y } \\
\text { HOSPITAL DE CLLINICAS) }\end{array}$ & $55,4 \%$ & $41,3 \%$ \\
\hline & $\begin{array}{l}\text { COBERTURA PRIVADA (IAMC y } \\
\text { seguros Privados integralas) }\end{array}$ & 33.25 & 40,65 \\
\hline & OTROS & $11,4 \%$ & $8.9 \%$ \\
\hline & TOTAL & $100 \%$ & $100,0 \%$ \\
\hline \multirow[t]{4}{*}{$\begin{array}{l}\text { INTERIOR } \\
\text { < 5000Y RURAL }\end{array}$} & $\begin{array}{l}\text { COBERTURA PÚBLICA (MS PIASSE y } \\
\text { HOSPITAL DE CLLAICAS) }\end{array}$ & $53,4 \%$ & $42,3 \%$ \\
\hline & $\begin{array}{l}\text { COBERTURA PRIVADA (IAMC y } \\
\text { Seguros Privados Integralas) }\end{array}$ & $38,4 \mathrm{~s}$ & 51,968 \\
\hline & OTROS & $8.2 \%$ & $5.8 \%$ \\
\hline & TOTAL & $100,0 \%$ & $100,0 \%$ \\
\hline
\end{tabular}

\section{RECURSOS FINANCIEROS (USD):}

- Para la cobertura sanitaria de la población rura incorporaida al SNIS, sumaron 360 millones 2008/2010. -Los costos de un equipo medico con las tres especialidades básicas para un servicio en el Primer Nivel de Atención de acuerdo con la norma 359/2007, sumaba para este trieño 270 mil.

- Si se considera la necesidad de construcción desde cero y equipamiento de un nuevo policlinico cumpliendo con la norma 416/2002, estimado en 100 mil, en el caso de los tres departamentos censados, hubiera implicado un gasto de 26,5 millones, pero como los ingresos superaron los 47 millones hubiera generado un resultado económico favorable a los prestadores privados por 19,5 millones.

- Teniendo en cuenta que no se cumplió con ninguna de las dos normas citadas, que las contrataciones han sido escasas y las inversiones en el Primer Nivel rural han sido prácticamente nulas, todo indica que ha sido un negocio de altisima rentabilidad para los prestadores privados.

RIFRAESTRUCTURA DE LAS POLICLIINICAS:

- El 10\% de la infraestructura básica está en estado regular o critico, 9 de cada 10 en ésta situación son rurales.

2 El $18 \%$ no cuenta con servicios básicos, todas son rurales.

- La infraestructura sanitaria es inadecuada en el $100 \%$ de los casos, peroel

$81 \%$ de las policlinicas comunitarias urbanas están en estado regular, mientras que el $57 \%$ de las policlinicas rurales están en estado crítico.

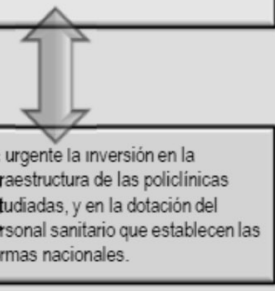

\section{Gráfico 6. Conclusiones}

La investigación, en particular por su metodologia, ha contribuido al proceso de priorización de la salud rural como política pública. La generación de evidencia sobre la situación, el facilitar el encuentro entre comunidades fuertemente aisladas, el generar ámbitos de intercambio de saberes encuentre entre las comunidades, la academia y el gobierno, la cogestión de jornadas de capacitación, el apoyo a la formulación de acción de promoción de salud en las comunidades y acciones de lobby, son algunas de las actividades que se han ejecutado.

\section{la ESCASA PROBLEMATIZACIÓNY REFLEXIÓN}

en relación los conceptos de salud y de Atención

Primaria de Salud

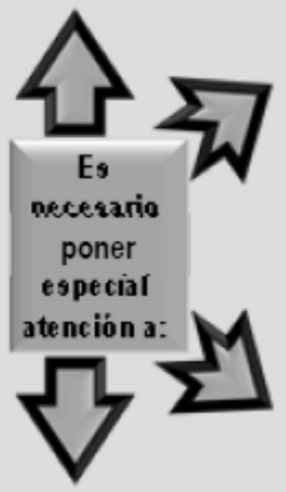

Ia ACCESIBILIDAD, como indicador clave entre las dimensiones que afectan el cumplimiento del principio de universalidaden la salud. Considerando aspectos geográficos, económico-financieros, organizacionales y/o burocráticos, culturales y/o simbólicos, y legales o normativos

Ia INTEGRALIDAD de las políticas públicas previstas por la estrategia de APS, la reforma tal como está planteada hasta el momento, no es suficiente para lograr la Atención Primaria en Salud Integral.

\section{Una señal positiva}

\section{PROGRAMA NACIONAL DE SALUD RURAL}

Existen anuncios desde la autoridad sanitaria en relación a la implementación de un plan de salud rural y a que el tema es prioridad para este equipo de gobierno. Para ello es necesario consolidar el desarrollo del primer nivel de atención en el medio rural, y ello (en I propuesta gubernamental) implica:

- Canasta Básica de prestaciones que deberán ser realizadas en el propio territorio

- Distancias máximas ente los servicios y el domicilio de los usuarios

- Infraestructura mínima, equipamiento y RRHH

- Obligatoriedad de las rondas preventivas
IOS DETERMINANTES SOCIALES DE LA SALUD-

discriminadas por regiones -, no son suficientemente incorporadas en la definición de políticas públicas que garanticen el derecho a condiciones de vida y de atención a la salud de manera continua y universal, de acuerdo al principio de equidad incluyendo residencia geográfica, nivel de instrucción, género, generaciones, ocupacional-
Será necesario, incrementar las alianzas con socios nacionales y organizaciones que incidan mediante sus acciones en las definiciones de políticas sanitarias, para lo cual, será necesario también contar con socios internacionales en la continuidad de la construcción de estrategias que habiliten a nivel del pais profundizar en la APS Integral. 
prestadores privados ante el silencio del sector público, ingresa a un servicio de salud idealizado en el imaginario social por su confort, hotelería y calidad de servicios. Sin embargo, a la hora de efectivizar el uso aparece la desilusión por no poder acceder a los beneficios prometidos, fundamentalmente por razones de accesibilidad geográfica-física, que resulta clave en el medio rural (véase el apartado a4) del capítulo de metodología). Los problemas de la accesibilidad económico-financiera (asequibilidad) dan cuenta de una población de escasa capacidad adquisitiva para hacer frente a los gastos de los servicios sanitarios cuando estos no se brindan de manera integral. Es en el sector privado donde ha crecido más la nómina de afiliados, mientras que las policlínicas comunitarias y rurales utilizan los escasos recursos materiales brindados por el sector público (fundamentalmente ASSE). Los acuerdos de complementación (publico-privado, privado-privado, público-público) entre prestadores no están establecidos con claridad, lo que genera que durante la espera de su definición quien asume los costos es mayoritariamente el prestador público, dado que posee una red de primer nivel mayormente desconcentrada (véase gráfico 4). La accesibilidad legal se refiere al marco normativo que se da al derecho a la salud en el país, en el que el primer gobierno de izquierda anuncia la formulación de la normativa que reconoce por primera vez explícitamente a la salud como derecho y bien público. Sus aspectos organizacionales y burocráticos refieren a la organización interna de los servicios para brindar atención a la población. Se ha detectado que frente a las falencias institucionales, este papel es asumido por la comunidad como organizador de la logística: turnos, horarios, recorridos, lista de espera, disponibilidad de recursos, difusión de información, etc. Estas tareas se realizan sin apoyos, de forma honoraria y en muchos casos invirtiendo recursos propios para que "esto siga". Muchas veces se verifica que la realización de estas tareas es la forma de participación comprometida que ha desarrollado la comunidad, que en muchos casos 'es una tarea invisibilizada o francamente desvalorizada'. Asimismo, a tres años de implementada la ley, en los servicios de salud mayoritariamente aún no se consolidan espacios de participación de usuarios que sean posibles, saludables, confiables, crítico-constructivos y que se hallen integrados a la institucionalidad. Frecuentemente, esos espacios de participación (cuando se intentan) son vividos persecutoriamente por prestadores y usuarios. Desde el punto de vista de los usuarios se explicita miedo a la hora de opinar, mucho más a la hora de denunciar. Estos miedos básicos (a la pérdida y al ataque) coartan una real apropiación de derechos en que la capacidad de organización y movilización se ven apaciguadas, ya que la capacitación e información, la crítica, la denuncia, la exigencia, son vistas como acciones que pueden generar represalias. La metodología de los Encuentros-Foros ha resultado exitosa en varios aspectos: a) permitió a las organizaciones aliadas su desarrollo organizativo; b) puso en contacto cara a cara a las autoridades sanitarias con la población de las comunidades geográficamente más alejadas; c) alivió la sensación de olvido, abandono y desesperanza de varias comunidades rurales, conectándoles con semejantes y facilitando el intercambio y la cooperación horizontal.

Las comunidades se muestran en la integralidad de sus necesidades de espacios, de tiempos de recreación y de encuentro, de llegada de mejoras y oportunidades. Estas necesidades, cuando se transforman en propuestas estatales para la salud del medio rural, corren el riesgo de reducirse a un paquete de servicios básicos y esporádicos que no contemplan esta diversidad de posibles intervenciones con una mirada integral inter y transdisciplinaria. Si esto ocurriera, al no realizarse la necesaria discriminación positiva para aquellos que están en peores condiciones de acceso, no se podría hablar del cumplimiento del principio de equidad, que es uno de los pilares básicos del SNIS. Seguramente, será necesario incrementar un proceso de alianzas con socios directos y organizaciones que incidan mediante sus acciones reivindicativas en estas definiciones, para lo cual será necesario también contar con socios internacionales en la continuidad de la construcción de estrategias que habiliten a nivel del país a profundizar la APS-I que las leyes de creación del SNIS proponen y este equipo de investigación intenta contribuir a concretar. 


\section{Referências}

ASOCIACIÓN LATINOAMERICANA DE MEDICINA SOCIAL (ALAMES); CENTRO BRASILEIRO DE ESTUDOS EM SAÚDE (CEBES). Documento de posición conjunto. 2011. Disponible en: <http:// www.alames.org/documentos/alamescebesrio.pdf> Acceso en: 10 feb. 2012.

ALMEIDA-FILHO, N. Epidemiología sin números. WDC: Organización Panamericana de la Salud, 1992. (PALTEX, 28).

La ciencia tímida: ensayos de desconstrucción de la epidemiología. Buenos Aires: Lugar, 2000.

ANDER-EGG, E. Repensando la Investigación-Acción Participativa. 4. ed. Buenos Aires: Lumen Humanitas, 2003.

AROUCA, S. El Dilema Preventivista. Contribuciones a la compresión y crítica de la Medicina Preventiva. Buenos Aires: Lugar Editorial, 2008. (Colección Salud Colectiva).

ASTORI, D. Astori Presidente: primeras 30 medidas de gobierno del Frente Amplio. 2009. Disponible en: <http://www.2121.org.uy/ noticias/8-comunicados-de-prensa/1280--primeras-30-medidasde-gobierno>. Acceso en: 10 feb. 2012.

BORGIA, F. ¿Cambiando la herencia de los gobiernos postdictadura? Los primeros pasos... hacia la utopía concreta: La mirada puesta en la indigencia la inequidad en salud. 2005. Dissertação (Mestrado em Sociología) - Universidad de la Republica, Montevideo 2005.

¿Cuál es la relación entre la Salud y los Derechos Humanos?: Derechos Humanos en el Uruguay - Informe 2005. Montevideo: SERPAJ, 2005.

La salud en Uruguay: avances y desafíos por el derecho a la salud a tres años del primer gobierno progresista. Revista de Medicina Social, Nova York, v.3, n.2, 2008.

La Salud en Uruguay: Avances y desafíos por el Derecho a la Salud en las vísperas del cuarto año del primer gobierno progresista. Derechos Humanos en el Uruguay - Informe 2008. Montevideo: SERPAJ, 2008.

El derecho a la salud en Uruguay: luces, sombras, esperanzas y desafíos. Derechos Humanos en el Uruguay Informe 2009. Montevideo: SERPAJ, 2009.

BREILH, J. Epidemiología Crítica: ciencia emancipadora e interculturalidad. Buenos Aires: Lugar Editorial, 2003. (Colección Salud Colectiva).

Epidemiología: economía política y salud. Quito: Universidad Andina Simón Bolívar - Corporación Editora Nacional, 2010.

CAPÍTULO URUGUAY - PIDHDD. Informe Alternativo al Comité DESC-ONU. Montevideo: Capítulo Uruguay - PIDHDD, 2010.
CEBES. Determinação Social da Saúde. Saúde em Debate. Rio de Janeiro, v.33, n.83, set/dez. 2009.

CETRULO, R. (Comp.). Orlando Fals Borda, Carlos Rodrigues Brandao: Investigación Participativa. 2. ed. Montevideo: Instituto del Hombre - Ediciones de la Banda Oriental, 1987.

FALS BORDA, O.; RAHMAN, M. A. Acción y conocimiento: como romper el monopolio con Investigación - Acción - Participativa. Santafé de Bogotá: CINEP, 1991.

FREIRE, P. Pedagogía de la esperanza. Un rencuentro con la pedagogía del oprimido. Madrid: Siglo XXI, 1993.

FRENTE AMPLIO. V Congreso Extraordinario: Zelmar Michelini, 13 y 14 de diciembre de 2008. Montevideo: Casa Líber Seregni - Frente Amplio, 2008.

Plataforma Electoral: Propuestas para seguir construyendo un país de primera. Mujica-Astori, un gobierno honrado, un país de primera. Montevideo: Frente Amplio, 2009.

GARCÍA, J.C. La medicina estatal en América Latina. Revista del Instituto Juan César García. A.1, n.1, jan. 1991.

Pensamiento social en salud en América Latina. México: Interamericana- OPS/OMS, 1994.

GRANDA, E. Práctica en Salud y Socialismo: elementos para la reflexión. In: Edmundo Granda Ugalde: La Saluda y la vida v.1. Quito: Ministerio de Salud Pública de Ecuador - Consejo Nacional de Salud - Universidad de Cuenca - Universidad de Loja - ALAMES - OPS/OMS, 2009.

GUERRERO, B.; GARCÍA, J.; SÁNCHEZ, B. Las Ciencias Sociales y la invención del tercer mundo: a propósito de la obra académica de Orlando Fals Borda. Revista Historia de la Educación Latinoamericana. v.12, 2009, p. 42-61.

JUNTA NACIONAL DE SALUD (JUNASA). Rendición de Cuentas. Ejercicio 2008. Disponible en: <http://www.msp.gub.uy>. Acceso en: 15 jan. 2011.

JUNTA NACIONAL DE SALUD (JUNASA). Rendición de Cuentas. Ejercicio 2009. Disponible en:< http://www.msp.gub.uy>. Acceso en: 15 jan. 2011.

KUHN, T. La estructura de las revoluciones científicas. Madrid: Fondo de Cultura Económica de España, 2005.

LAURELL, C., NORIEGA, M. La Salud en la fábrica, México: Era, 1989.

LAURELL, C. La reforma contra la salud y la seguridad social. México: Era, 1997. 
MUÑOZ, M. J. Conferencia de la Ministra de Salud Pública en la Reunión Sub-Regional de Representantes y Directores de Centro del Cono Sury Área Andina. WDC, 13-17 mar. 2006.

NOGUEIRA, N.P. (Org). Determinação Social da Saúde e Reforma Sanitária. Rio de Janeiro: CEBES, 2010.

OLESKER, D.; Conferencia: El Sistema Nacional Integrado de Salud. Ginebra: OIT, 2008.

ORGANIZACIÓN MUNDIAL DE LA SALUD (OMS). Subsanar las desigualdades en una generación: Alcanzar la equidad sanitaria actuando sobre los determinantes sociales de la salud. Ginebra: OMS, 2008.

- Cerrando la brecha: La Política de acción sobre los determinantes sociales de la salud. Río de Janeiro: OMS, 2011.

PAULINO, C.; PEREDA, M. La pobreza Rural en el Uruguay: situación actual y aportes para el diseño de una estrategia para su combate. Montevideo: FIDA, 2008.

PILÓN, J. El Sistema Nacional Integrado de Salud (SNIS) y el Seguro Nacional de Salud (SNS), Montevideo: Unión de la Mutualidad del Uruguay - Uruguay Saludable/MSP, 2009.
RIELLA, A.; MASCHERONI, P. Población, ingresos y hogares agrodependientes. En: ANUARIO 2009. Montevideo: OPYPA, 2009.

ROJAS-OCHOA, F., MÁRQUEZ, M. (Comp.). ALAMES en la memoria. Selección de lecturas. La Habana: ALAMES Cuba/Editorial Caminos, 2009.

SAMAJA, J. Epistemología y Metodología. Elementos para una teoría de la investigación científica. Buenos Aires: Eudeba, 2003.

SINDICATO MÉDICO DEL URUGUAY. Disponible en: <http://www. smu.org.uy/sindicales/comunicados/laudos.htm>, Acceso en: 20 feb. 2012.

STOLKINER, A. et al. Reforma del Sector Salud y utilización de servicios de salud en familias NBI: estudio de caso. En:DOMINGUEZ, M. A. et al. La Salud en Crisis- Un análisis desde la perspectiva de las Ciencias Sociales. Buenos Aires: Dunken, 2000.

Recebido para publicação em Abril de 2012

Versão definitiva em Agosto de 2012

Suporte financeiro: Iniciativa de Investigación en Salud Mundial

Conflito de interesse: inexistente 